\title{
An Analysis on English to Chinese Translation of Electrical Engineering Texts from the Perspective of Professionals under the Guidance of Skopos Theory
}

Xiaoli Song, Zi Ye

Lecturer, Department of Foreign Languages, North China Electric Power University, Beijing, China

Corresponding Author: Xiaoli Song, Zi Ye, E-mail: flyivy@163.com

\section{ARTICLE INFORMATION}

Received: August 10, 2020

Accepted: September 15, 2020

Volume: 3

Issue: 9

DOI: 10.32996/ijllt.2020.3.9.18

\section{KEYWORDS}

Translation; Electrical

Engineering; Skopos Theory;

Semi-structured Interview

\section{ABSTRACT}

With the Skopos Theory as the theoretical foundation, this study aims at analyzing the specialized translation of electrical engineering texts from the professionals' perspective. To do this, we first conducted a questionnaire survey to collect the information on the professionals' needs and requirements on the translation of English texts in the realm of electrical engineering. Then, we selected an English academic paper published in a prestigious journal in this field and conducted a semi-structured interview on the Chinese translation of this paper with three professionals in this field. Based on the results of both questionnaire and interview, we analyzed the translation of electrical engineering texts from English to Chinese at lexical, syntactic and textual levels with the guidance of Skopos Theory. It is found from the questionnaire survey that electrical engineering professionals in China do have a need for translation of English texts in this field, and they emphasize the accuracy of terms and information integrity of the target text. From the interview, we also find that Skopos Theory has a strong guiding effect on the specialized translation of electrical engineering texts. Under the guidance of this theory, translators can adopt various translation strategies to enhance their translation output and optimize its communicative effect.

\section{Introduction}

Thanks to the development of globalization in recent years, communications in the fields of politics, culture and technology are becoming increasingly frequent worldwide. In China, the vigorous development of the electric power industry and the application of new electric power technology calls for exchange and cooperation with other countries. World widely, the majority of the latest research and technological development is published in English. Nevertheless, for most Chinese professionals studying or working in the electrical engineering field, due to the language insufficiency, reading original English texts does pose difficulties on understanding and reading speed. Therefore, the translating of English electrical engineering texts plays an important role in promoting and spreading the latest technological development in China, thus demanding more and more translators to join in.

Since the late 1970s, researchers started to apply the functionalist approach to translation studies, based on which Vermeer (1987) put forward the "Skopos Theory". "Skopos" is the Greek word for "aim" or "purpose". According to Vermeer (1989), translation aims to "produce a target text in a target setting for a target purpose and target addressees in target circumstances." He also proposed three rules to guide the translating, namely the skopos rule, the coherence rule and the fidelity rule, among which the skopos rule is the leading one (Vermeer,1989).

Although the significance of the specialized translation of electrical engineering texts is evident, there are few researches on it. This gap in the literature has served as a point of departure for the present study, which takes the Skopos Theory as the theoretical framework and explores the translation in the field of electrical engineering from English to Chinese from the perspective of professionals in this field. Two questions will be focused on: 1) What are the needs and requirements of the professionals on the Chinese translation of English texts in electrical engineering field? 2) Based on the professionals' needs

K C AL-KINDI CENTER

$\mathbf{R}$ D FOR RESEARCH AND $R$ D DEVELOPMENT Your gateway to world-class research
Published by Al-KindiCenter for Research and Development. Copyright (c) the author(s). This is an open access article under CC BY license (https://creativecommons.org/licenses/by/4.0/) 
and requirements, what translation strategies can be adopted in the translating process under the guidance of Skopos Theory?

\section{Features of Electrical Engineering Texts}

Texts in the field of electrical engineering have their own features lexically and syntactically. Firstly, it includes a large number of terms. It is worth noting that some terms are only used in electrical engineering context, while others can be used in other contexts as well, but with quite different meanings. Terms like "impedance", "circuit breaker" and "inductive reactance" fall into the first type. Terms like "utility" and "slip" belong to the second category: "utility" means "the quality of being useful" in daily life, but in electrical engineering context, it means "customer"; "slip" means "slide or fall" in daily life, but in electrical engineering context, it means "the angle speed difference between rotor and stator". The second feature is the frequent use of abbreviations, which are simple in expression, convenient in spelling, and easy to read and understand. This contributes to the conciseness of electrical engineering texts. Examples are VSC (Voltage Source Converter), MMC (Modular Multilevel Converter), and DFIG (Doubly Fed Induction Generator). Thirdly, professional English in electrical engineering is featured as using declarative sentences with present tense (Tu \& Jin, 2002). Since electrical engineering texts focus on narration, reasoning and argumentation of objective things, passive sentences are also frequently used to avoid subjectivity.

\section{Skopos Theory}

\subsection{Development of Skopos Theory}

The functionalist approach in translation emerged in the late 1970s in Germany. Thereafter, it has developed to be one of the most influential theories in the realm of translation studies. "Functionalism", was defined by Christiane Nord as "focusing on the function or functions of texts and translation" (Nord, 2001). She further stated that "Functionalism is a broad term for various theories that approach translation in this way. Besides, Nida's theory of dynamic/functional equivalence also contributed greatly to functionalism in translation. Dynamic equivalence viewed translation in a communicative way, which is against the traditional linguistic understanding of translation as a code-switching process. According to Nida, "translating consists in reproducing in the receptor language the closest natural equivalent of the source-language message, first in terms of meaning and secondly in terms of style" (Nida \& Taber, 2004).

Based on the functionalism in translation studies, the Skopos Theory was finally developed with the endeavors of the following three outstanding functionalists: Katharina Reiss, Hans J. Vermeer, and Christiane Nord. Reiss found that, in some cases, equivalence was not necessary. She claimed that, the function of the target text (TT), rather than the source text (ST), should be considered as the dominant factor. Reiss believed that "text typologies help the translator specify the appropriate hierarchy of equivalence levels needed for a particular translation Skopos" (Nord, 2001). So, she categorized texts into three types, namely informative text, expressive text and operative text. Translation methods and strategies should be adopted in line with the text type. An informative text is content-focused, aiming to deliver information to its readers. Translating informative text requires the translators to keep the target text plain and logical. An expressive text demands the translators to translate the source text into elegant target text so as to produce "an analogous stylistic effect" (Nord, 2001). An operative text is appellative-focused, appealing to target readers to act in a certain way. Reiss put forward that translators should apply the adaptive method to create equivalent effects for target text readers. Reiss's text typology laid the groundwork for the Skopos Theory. Peter Newmark shared a similar view with Reiss, and he further explained that "few texts are purely expressive, informative or vocative: most include all three functions, with an emphasis on one of the three" (Newmark, 1998).

Following Reiss's studies of translation, Vermeer (1987) proposed that the function of both the source and target text are the dominant considerations in translating and interpreting. This idea was first named as "Skopos Theory". "Skopos" is the Greek word for "aim" or "purpose". According to Vermeer (1989), translation aims to "produce a target text in a target setting for a target purpose and target addressees in target circumstances." As a result, the purpose or skopos of the translation eventually shapes the way of the target text and determines translators' adoption of methods and strategies (Vermeer, 1989). Nord then distinguished the purposes into three possible kinds: the general purpose of the translator in the translating process, the communicative purpose of the target text in the target situation and the purpose of a specific translation strategy or procedure (Nord, 2001). Among the three purposes, the communicative purpose of the target text is emphasized in Skopos Theory. In order to guide the translation process, Vermeer further proposed three rules, i.e. the skopos rule, the coherence rule and the fidelity rule, among which the skopos rule is the leading one (Vermeer,1989).

Although Nord shared similar views on the functionalist approach to translation studies with the two pioneers mentioned above, she regarded translation as a purpose-focused intercultural communicative activity between individuals instead of by the translator only. To perfect the Skopos Theory, Nord indicated that "the concept of 'loyalty' should be added to the 
framework of functional theories", which is called "function plus loyalty" translation model (Nord, 2001). By integrating loyalty into Skopos Theory, the translator has the obligation to be responsible for the participants in the translation project, as well as coordinate whenever contradictory intentions arise.

\subsection{Three Rules of Skopos Theory}

\subsubsection{Skopos Rule}

Skopos rule is the core and primary principle of Skopos Theory, which determines the translation activity. This rule comprises the purpose of the source text, the communicative purpose in the target culture, and the translation strategies to achieve the equivalent purpose. According to Nord, the purpose has three categories: "the general purpose, the communicative purpose and the purpose of a particular strategy or procedure" (Nord, 2001). In the translating process, translators should "translate/ interpret/ speak/ write in a way that enables your text translation to function in the situation it is used and with the people who want to use it and precisely in the way they want it to function" (Nord, 2001). If the cultural background is changed in the target language culture, some norms can be adjusted to fit the cultural background and criterion of the target settings. Because of the change of settings, the target text may not be equivalent to the source text, for the priority of translation is to achieve the purpose of communication from the perspective of target audience.

\subsubsection{Coherence Rule}

Coherence rule means that translation should be acceptable as long as it conforms to the recipient's situation (Reiss and Vermeer, 2014). The translation should be consistent with the expectation of the target audience so that the readers can understand the target text and it should make sense in the communicative scenario and culture in which it targets (Nord, 2001). Schäffner (1998) pointed out that the target text must be sufficiently coherent so that the target user can understand it because they have assumed background knowledge and situational context. The coherence rule of Skopos Theory refers to intratextual coherence, namely the coherence within the language, which enables the target text understandable and acceptable by the target audience from the target culture. Thus, intratextual coherence works as a bridge connecting the source text and the target audience.

\subsubsection{Fidelity Rule}

The fidelity rule refers to intertextual coherence between target text and source text, namely the relationship between source text and target text, which is subordinate to coherence rule and skopos rule. It requires the translation to be faithful to the source text, be consistent in meaning and style. Nida and Taber (1982) stress the importance of being faithful to the ST. Chesterman (2001) states that a translator should be like a mirror that reflects the ST. Depending on the translators' comprehension of the source text and the aim of the target text, fidelity may take different forms and be in varying degrees (Fan \& Zhongying, 1990).

In translating process, the three rules are considered different in significance. However, "the three rules interact with each other. (Bian, 2008). Basically, the coherence and fidelity rule are subordinate to the skopos rule, and the fidelity rule is subordinate to the coherence rule. "If the translation skopos requires a change of function, the standard will no longer be inter-textual coherence with the source text. If the translation skopos demands intratextual incoherence (as in the theatre of the absurd), the standard of intratextual coherence is no longer valid" (Nord, 2001). Similarly, Vinay and Darbelnet (1995) argue that faithfulness should not be achieved all the time.

\section{Methodology}

This study uses questionnaire survey and semi-structured interview as the research methods. Questionnaire survey provides the quantitative basis to the study while a semi-structured interview offers the qualitative data for analysis. It is worth noting that in this study, the interview is the main research method, while the questionnaire survey is a supplementary one.

\subsection{Questionnaire Survey}

In this study, we first conduct a questionnaire survey to get the quantitative results. In the questionnaire, 7 questions are designed to obtain the professionals' needs and requirements on the Chinese translation of English texts in the electrical engineering field. The questionnaire are sent to 130 people studying or working in the field of electrical engineering for more than five years, who are qualified to be called professionals. In the end, 123 people send back their completed questionnaires. As Figure 1 shows, among the 123 respondents, 39\% are postgraduates majoring in electrical engineering, $22 \%$ are university professors, $14.6 \%$ are working in the scientific research institutions, and $24.4 \%$ are working in the electrical engineering corporations. 


\section{Career Background of the Participants in the Questionnarie Survey}

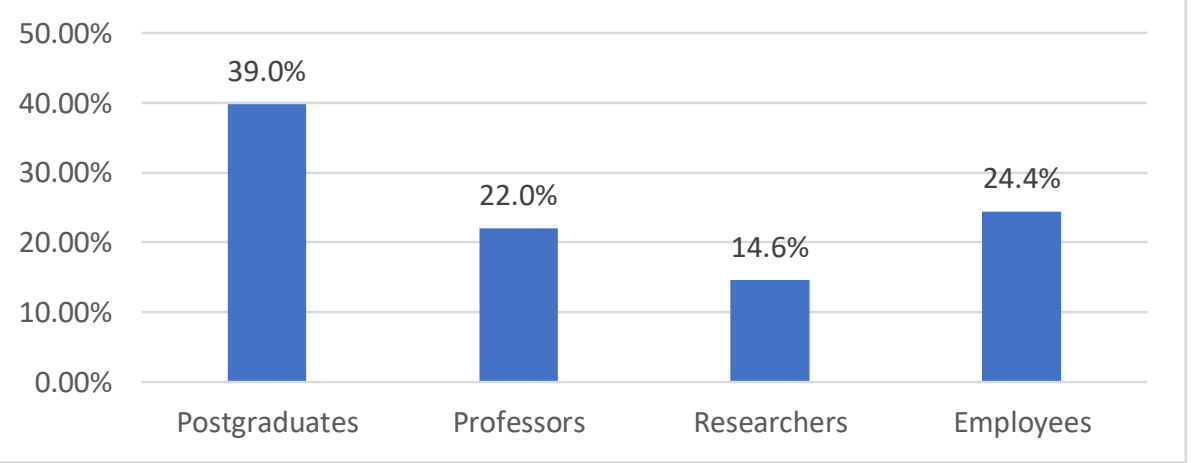

Figure 1. Career Background of the Participants in the Questionnarie Survey

\subsection{Semi-Structured Interview}

Then, an online interview with three participants is conducted by the researchers themselves. The interview is a semistructured one, in which an interview schedule is used, with more open-ended questions. Closed questions are related to the accuracy of terms and translation preference on abbreviations in the selected translation material while open questions are related to the logic analysis of sentences and paragraphs in the selected translation material as well as suggestions on the translation of English electrical engineering texts. Through combining the closed and open questions, the interview can better motivate the participants to fully engage in the interview, contributing to the co-building of knowledge.

\subsubsection{The Participants}

The three participants in the interview are all postgraduates in a prestigious university in China, majoring in electrical engineering. They have been studying this discipline for five to six years, and therefore, can be regarded as professionals. Meanwhile, they all have passed Chinese College English Test 6, the most popular English test for Chinese college students. Therefore, they can be called competent English users. In their studies, more than 40 percent of their academic reading is in English. Being familiar with both English and Chinese text in this field, the participants are believed to be able to give some constructive suggestions on the translation of English electrical engineering texts. In the interview, the participants are not anonymous. Saldanha and O'Brien (2014) reports that "anonymity is not necessarily a requirement or an advantage, particularly in unstructured individual interviews, in cases where the participants may have been chosen because the information sought can only be provided by them and not someone else."

\subsubsection{The Selected Translation Material}

In the research, we selected an English electrical engineering text and its Chinese version (translated by the authors) as material for both questionnaire survey and interview. The source text is an academic paper, titled "Definition and Classification of Power System Stability". It is published on the 19th issue of IEEE Transactions on Power Systems in 2004, a top journal of electrical engineering. It not only summarizes the cognition of academia and industry on this issue in the 20th century, but also pioneers the power system stability research in the 21st century and establishes the research guidelines. Prabha Kundor, the author of this paper, is an expert on power system stability control.

It is necessary to have a clear understanding of the text type of the source text because it determines the choice of translation methods. According to Reiss' text typology, the source text selected in the study belongs to informative type, with information delivery as its main function. For informative text, the target text is judged by maintaining the accuracy, practicability, conciseness and logicality of the source text.

\subsubsection{The Procedure of the Interview}

In the interview, some background questions are asked first, such as the major of the participants, their frequency of reading English texts in the field of electrical engineering, and their preference of original English version or Chinese translation version. Then, the participants are asked what they emphasize most about the translation of English electrical engineering texts. Thirdly, questions related to the translation of the selected text at lexical level, syntactic level and textual level are discussed in detail with the participants. For example, how to handle the abbreviations in the selected translation material; whether the terms in the selected translation material are translated accurately; whether the sentences in the target text deliver the exact meaning of the source text; whether some translation strategies improve the quality of translation from the 
professionals' point of view. After that, open questions about the logic analysis of sentences and paragraphs in the selected translation material are asked. In the end, we invite the participants to offer some suggestions on the translation of this type of text, particularly for translators who are not professionals in the electrical engineering field.

\section{Result Analysis}

\subsection{Analysis of Questionnaire Survey Results}

Based on the data collected from the questionnaire, which can be shown in Figure 2, we have found that, for $64.22 \%$ of respondents, $20 \%--50 \%$ of their academic reading is in English. For $18.7 \%$ of respondents, English papers account for $50 \%--$ $70 \%$ of their academic reading. These data show that, professionals do have a need to read English texts in this field. When it comes to the major difficulties in reading English texts, the top three are lack of vocabulary, poor English reading comprehension capability and low reading speed. Moreover, among the 123 respondents, $74 \%$ choose to read the Chinese translation of the English texts if available. This information indicates that professionals have a strong demand for the translation of English texts in the electrical engineering field.

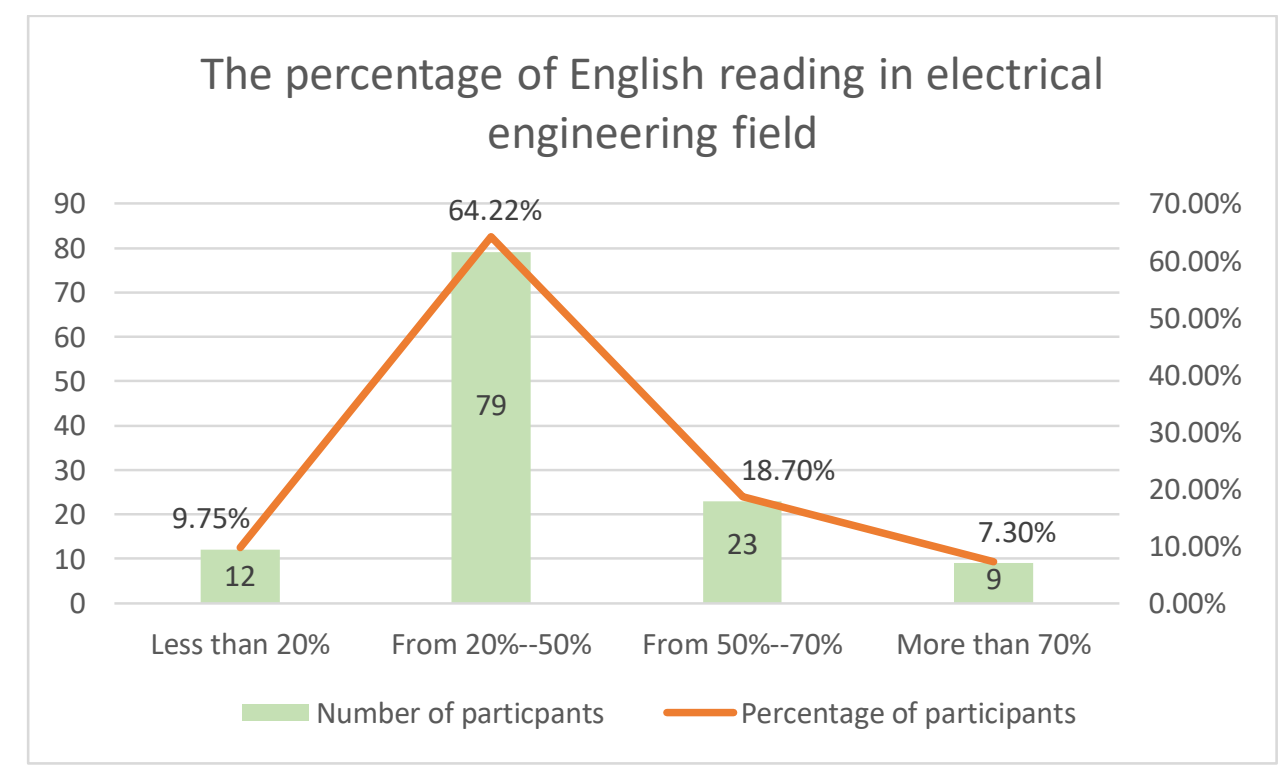

Figure 2. The percentage of English reading in electrical engineering field

At the end of the questionnaire, the respondents are asked to prioritize the importance of translation quality factors (accuracy of terms, information integrity, logic, coherence, translation style). It is shown from the data that accuracy of terms is of the greatest importance for professional readers, then followed by information integrity and logic of the target text. The least important factor pointed out by the respondents is the translation style. In other words, the Chinese professionals in the electrical engineering field emphasize more on the accuracy of terms, logic and information integrity when reading the translation of English texts.

\subsection{Analysis of Interview Results}

In the interview, both closed and open questions are put forward, discussing the translation of the selected electrical engineering text --- Definition and Classification of Power System Stability, at lexical, syntactical and textual levels. Based on the Skopos Rules, we analyze the feedback from the participants, exploring the specialized translation of electrical engineering at these three levels. Moreover, suggestions given by the participants on translating English texts in this field are summarized in this part. 


\subsubsection{Translation at Lexical Level}

\section{Translating Abbreviations}

Example 1.

Source Text (ST): The problem of defining and classifying power system stability has been addressed by several

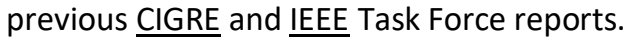

Target

(TT): 之前的CIGRE（国际大电网会议）和IEEE（国际电气与电子工程师协会）工作组报告已经解决了电力系统稳定性

的定义和分类问题。

\section{Example 2.}

ST: Voltage stability problems may also be experienced at the terminals of HVDC links used for either long distance or backto-back applications.

\section{TT: 用于长距离或背靠背应用的HVDC（高压直流输电）线路的端口也可能遇到电压稳定性问题。}

An important lexical feature of professional texts in electrical engineering is the frequent use of abbreviations. For the source text, the use of abbreviations helps to maintain the conciseness of the text so as to save the time of reading. Under the skopos rule, this communicative purpose should be maintained in the target text. In the interview, Zhang, Yao, and Chen all agree that the abbreviations such as IEEE、CIGRE、HVDC should be kept since these abbreviations are also familiar to the Chinese professionals and it can save the reader's time.

\section{Translating Terms}

As the questionnaire results show, the accuracy of terms is the most important translation quality factor for the professionals. Since the translation material discussed is an academic paper in the field of electrical engineering, there are many terms throughout the whole text. Based on the reflections of the interview participants, the translation of terms is discussed as follows.

Example 3.

ST: For instance, a fault on a critical element followed by its isolation by protective relays will cause variations in power flows, network bus voltages, and machine rotor speeds

$\Pi$ : 例如, 一个关键元件发生故障, 然后通过继电保护装置进行隔离, 这将导致潮流、网络母线电压和电机转子速度 的变化。

Example 4.

ST: For example, two systems may both be stable with equal stability margins, but one may be relatively more secure because the consequences of instability are less severe.

TT: 例如, 两个系统可能都具有相同的稳定裕度, 若这两个系统均失稳, 造成的后果较轻的系统具有更高的安全性。

Example 5.

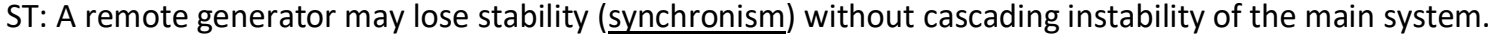

$\Pi$ T: 远端的发电机自身可能会失去稳定性（失步），而不会发生级联造成主系统的失稳。

Examples 3-5 are all technical terms in the field of electrical engineering. The Chinese version of "power flows "and "stability margins "can be found directly from the dictionary. In example 5, "synchronism" is translated into"同步” in the dictionary. But to make target readers understand the meaning better, we suggest combining the meaning of "lose" and "synchronism “together and translate this term into “失步”. In the interview, participants all agree that “失步” facilitates their comprehension. For this category of terms, often used in electrical engineering text only, direct translation is always the preferred strategy. However, translators are advised to take the context into consideration, and enable the translation to better serve the communicative purpose. 
Example 6.

ST: The trajectories that exit this desired region may either lead to structural changes (e.g., breaker tripping in a power system)

$T$ : 离开该区域的轨迹可能导致结构变化（例如，电力系统中的断路器跳闸）

Example 7.

ST: Depending on the network topology, system operating condition and the form of disturbance, different sets of opposing forces may experience sustained imbalance leading to different forms of instability.

$\mathrm{TT}$ : 根据电网拓扑结构、系统工况和扰动形式，不同的反作用力之间可能处于持续的失衡状态。

Example 6 and 7 are terms which are not only used in the electrical engineering field, but also in daily life. In example 6, "trip" is translated into “旅行" in most daily conditions, but Chen mentions that in the text of electrical engineering, it means “短路/跳闸”. In example 7, “network" was first translated as “网络”. Zhang explains that it is more acceptable to relate network/grid to “电网” in an electrical engineering text. For this type of terms, translators are advised to translate them into the technical terms according to the contexts. Otherwise, it will prevent the Chinese readers from the correct understanding of the text.

Example 8.

ST: The overvoltages that result when generator load changes to capacitive are characterized by an instantaneous rise at the instant of change followed by a more gradual rise.

$\pi$ : 当发电机负荷变为容性时, 产生的过电压的特征是电压在负荷变化瞬间会突变, 之后逐渐缓慢上升。

In example 8 , by adopting the translation strategy of deletion, the phrase "an instantaneous rise at the instant of change" is translated simply into“突变”. The meaning of “an instantaneous rise” is deleted here. According to Yao,“突变”is very concise and more acceptable for professionals in electrical engineering. So, translators should filter the information in the source text, reserve whatever is useful and delete whatever is not informative for a better understanding to the target readers.

Example 9.

ST: Small-disturbance rotor angle stability problems may be either local or global in nature.

TT: 小扰动转子角度稳定性问题本质上可以是李雅普诺夫意义上的局部稳定性问题或全局稳定性问题。

In example 9, “local or global problems" can be translated literally as “局部或全局问题”. However, Zhang explains that this may cause confusion because "local or global problems" may have both physical meaning and Lyapunov meaning. After discussing with his supervisor, he makes it clear that, according to the control theory of electrical engineering, "local or global problems" are associated with Lyapunov stability. Apparently, physical meaning of this phrase does not fits the context here. As a result, this part is translated into“李雅普诺夫意义上的局部稳定性问题或全局稳定性问题” by adopting the translation strategy of addition so that the target readers can comprehend this sentence more easily. This example shows, under Skopos rule, how addition serves the communicative purpose better.

Example 10.

ST: However, this problem can still occur when generators operate with constant excitation when subjected to the actions of excitation limiters (field current limiters).

$\mathrm{TT}$ : 然而, 当发电机受到励磁限制器（励磁电流限制器）的作用, 以恒定励磁运行时, 仍然会出现这个问题。

In example 10, Both Zhang and Yao point out that "field current" can be recognized easily by target readers as “励磁电流”. Therefore, the translation of “励磁电流限制器” is understandable to them. With this example, we find that in translating electrical engineering texts, it is feasible to apply the strategy of literal translation if no standardized translation version can be found. Professionals in this field are able to recognize the target the translator attempts to refer to. By adopting literal translation in professional papers in electrical engineering, communicative effect of the target text is successfully fulfilled. 


\subsubsection{Translation at Syntactic Level}

Fidelity rule requires that the translation should be faithful to the source text, being consistent in meaning. Since the purpose of translating academic text is to provide faithful information for the target audience, the requirement of fidelity tends to be quite high. However, on account of different sentence structures of English and Chinese, the forms of fidelity may vary. To achieve fidelity rule on syntactic level, two translation strategies are often applied, namely addition and literal translation.

Example 11.

ST: On a longer timescale, the effects of market structures may become prominent.

TT: 在更长的时间范围中, 电力市场结构的影响可能会变得突出（传统电力市场通常以天为时间单位）.

In example 11, the translation strategy of addition is adopted. The content of “（传统电力市场通常以天为时间单位）”is added to the original information. According to Chen, just like the stock market, the electricity market is measured by days. Therefore, it is better to add this explanation to enable the meaning of the target text more consistent with that of the source text.

Example 12.

ST: However, for assessing stability when subjected to a specified disturbance, it is usually valid to assume that the system is initially in a true steady-state operating condition.

TT1:

但是, 为了评估受到特定干扰时的稳定性, 通常 (忽略最初工况的小扰动并将系统的最初工况设定为稳态工作点。

TT 2: 但是, 为了评估受到特定干扰时的稳定性, 通常认为系统的初始状态在一个稳定的工况下。

In example 12, we first added “（忽略最初工况的小扰动）"，trying to explain that the steady-state operating condition can only be achieved when ignoring small disturbances. However, in the interview, both Zhang and Chen hold that professionals are clear that the initial operating condition is in a steady state with ignoring small disturbances and there's no need to add the explanation. Since target readers can understand the literal translation, there is no need to add explanation in this case. With the opinions of interviewees, we find that, in some cases, literal translation can achieve fidelity effect.

From example 11 and 12, it is shown that without a strong professional background, the translators may find it difficult in translating specialized texts. Apart from the traditional training of translation, translators are suggested to expand their background knowledge in the specialized field so that their translation work can better meet the needs of target readers.

\subsubsection{Translation at Textual Level}

Logic of the translation text is of importance to the professionals. In the questionnaire survey, logic ranks number 3 in terms of importance for the professionals. The coherence rule of Skopos Theory requires that the target text should be logical and acceptable by the target audience from the target culture. The intratextual coherence works as a bridge connecting the source text and the target audience. To achieve the coherence on textual level, elements in sentences and paragraphs should be reorganized in the structure of target language.

Example 13.

ST: Given the large size of power systems and the need to consider event-type perturbations that will inevitably lead to nonlinear models, it is clear that the task of determining stability of a power system will be a challenging one. It turns out, however, that our main tools in reducing the computational complexity will be our ability (and willingness) to utilize approximations, and the particular nature of event-type disturbances that we are analyzing.

$\mathrm{TT}$ : 鉴于大电力系统以及考虑故障型的扰动的需求, 这将不可避免地引入非线性模型, 因此, 确定电力系统稳定性将是一个具有挑 战性的任务。然而, 事实证明, 我们致力于用近似和对故障型干扰的特殊分析降低计算复杂性。

$\mathrm{TT} 2$ : 鉴于大电力系统以及考虑故障型扰动的需求（这将不可避免地引入非线性模型）, 显然, 研究电力系统稳定性将是一个具有 挑战性的任务。然而，事实证明，我们致力于用近似和对故障型干扰的特殊分析降低计算复杂性。

Example 13 has great logical complexity. In the translation process, the clause "that will inevitably lead to nonlinear models" is inserted into brackets because Zhang reflects that this will reduce the confusion of target text. Other modifications are made based on Yao's comment. She explains that in Chinese papers, it is more often to use “研究” rather than “确定”, even 
though the meaning of "研究" is a little bit far away from "determine". All the modifications tend to help the realization of the textual cohesion, enabling the target audience to understand the text better.

Example 14.

ST: Restored loads increase the stress on the high voltage network by increasing the reactive power consumption and causing further voltage reduction.

TT 1: 提高无功的需求以及进一步的电压损失，使储存的负荷功率对高压电网构成了极大的负担。

TT 2: 恢复的负荷会增加无功的消耗，无功消耗增大会导致电压下降，进而引起进一步的电压损失。

Although the above example sentence is not that long, the logic of the sentence is hard to handle in translating because of the differences in sentence structure between English and Chinese. In the interview, Zhang explains the logic in this way, "when there are some problems with the power system, the restored loads will increase the stress on the high voltage network because these loads will increase the reactive power consumption, and then result in the reduction of voltage. "Based on Zhang's explanation, we adjust the sentence order and rewrite the target text into the second version, which has better logical coherence. All the interviewees agree that the second version is more coherent and understandable.

Example 15.

ST: Some generators and loads may be disconnected by the isolation of faulted elements or intentional tripping to preserve the continuity of operation of bulk of the system.

$\mathrm{TT}$ : 一些正常工作的发电机和负荷可能因为故障元件隔离而断开, 或规避故障的影响故意跳闸, 以保持大系统工作的 连续性。

Example 15 describes two reasons of disconnection of generators and loads, one is the isolation of faulted elements, the other is the intentional tripping to preserve the continuity of operation. According to Chen's explanation, the isolation of faulted elements is the active disconnection to avoid the influence of faulted elements, and the intentional tripping is the passive disconnection. With Chen's account, we are more clarified about the reason of disconnection, and a better logical coherence is achieved in the target text, which works as a bridge connecting the target text and the target reader under coherence rule.

\subsubsection{Other Suggestions from Participants}

In the interview, the participants are invited to give some suggestions on translating English texts in the electrical engineering field. Zhang points out that logic of target text is of vital importance. Translators are suggested to adjust the order of sentences, if needed, to achieve a better logical effect. Yao illustrates that titles and subtitles of a paper should be translated correctly so that the reader can locate them easily. Translators should read more parallel Chinese papers in this field to familiarize with the writing pattern. Some parts of the text, such as tables and charts, conditions, and equations must be accurately translated. Chen points out that translators should pay more attention to the logic and coherence on syntactic and textual level. Small mistakes on lexical level can be neglected. Moreover, translators who do not have the professional background, are suggested to enlarge the knowledge reserve of electrical engineering. Since electrical engineering is a large discipline that has several subordinate disciplines, the translators should identify which discipline the translation material belongs to and translate them based on the exact discipline information.

\section{Conclusion}

In this study, we probe into the translation of electrical engineering texts under the guidance of Skopos Theory and reach the following conclusions. Firstly, the Chinese professionals in electrical engineering do have a need for translation of English electrical engineering texts. From their perspective, the accuracy of terms and information integrity of the target text are the most important aspects. Secondly, based on the analysis of interview results, we also conclude that functionalist perspectives are applicable to the translation of electrical engineering texts. The Skopos Theory prioritizes the purpose of translation and emphasizes the communicative effect of the target text. As a type of informative text, the electrical engineering texts aim to achieve communicative purposes so that the target audience (professionals) can have a better comprehension of the target text. Therefore, it can be concluded that Skopos Theory provides both theoretical and practical guidance to E-C translation of electrical engineering texts. Thirdly, under the guidance of Skopos Theory, translators can adopt translation strategies such as addition, deletion, and literal translation according to the purpose of the target text. Fourthly, translators are suggested to 
expand their background knowledge on electrical engineering so as to improve their translation output. We hope that this study could provide some perspectives and be of help to translators when translating electrical engineering texts.

In this study, interview is used as one of the research methods, which is rare to be seen in translation studies. However, all three participants of our interview are postgraduates studying in this field for more than five years. The single background of interview participants could be thought as the limitation of the study. In the future, in order to provide more convincing results and applicable suggestions on specialized translation in electrical field, we will further the study by including more participants with diverse social backgrounds, like research institutions, electrical power corporations and governmental originations.

\section{About the Author}

Xiaoli Song is a lecturer at North China Electric Power University. She holds a master's degree in Cross-Cultural Communication from University of International Business and Economics (Beijing). Her main research interests include translation and intercultural studies, Business communication across cultures.

Zi Ye obtains a bachelor's degree from North China Electric Power University and holds a master's degree in Translation and Interpreting from the University of Essex in 2020. Her research interest lies in translation, interpreting, and cross-cultural communications.

\section{References}

[1] Bassnett, S. (2002). Translation Studies (3rd ed.). London \& New York: Routledge.

[2] Bian, J. (2008). Inheritance and transcendence: a study of functionalist skopos theory of translation. Beijing: China Social Science Press.

[3] Chesterman, A. (2001). Proposal for a Hieronymic oath. The Translator, 7, 139-154.

[4] Deng, F., \& Deng, B. (2015). Research on teaching methods of English translation for electrical engineering. Education Teaching Forum, (51), 150-151.

[5] Edley, N., \& Litosseliti, L. (2010). Contemplating Interviews and Focus Groups. London \& New York: Continuum.

[6] Gentzler, Edwin. (1993). Contemporary Translation theories. London/New York: Routledge.

[7] Hickey, L. (ed.). (2001). The Pragmatics of Translation. Shanghai: Shanghai Foreign Language Educational Press.

[8] Laviosa, S.(1998). The Corpus-based Approach: A new paradigm in translation studies. London: Routledge.

[9] Munday, J. (2001). Introducing Translation Studies. London \& New York: Routledge.

[10] Newmark, P. (1998). Paragraphs on Translation. Clevedon: Multilingual Matters.

[11] Newmark, Peter .(2001). Approach to Translation. Shanghai: Shanghai Foreign Language Education Press.

[12] Nida, E. A., Taber, C. (1982). The Theory and Practice of Translation (2nd ed.). Leiden, The Netherlands: E.J. Brill.

[13] Nida, E. A., \& Taber, C. R. (2004). The Theory and Practice of Translation. Shanghai: Shanghai Foreign Language Education Press.

[14] Nord, C. (2001). Translating as a Purposeful Activity - Functionalist Approaches Explained. Shanghai: Shanghai Foreign Language Education Press.

[15] Pym, A. (2001). Introduction: The return to ethics in translation studies. The Translator, 7, 129-138.

[16] Reiss, K. (2000). Translation Criticism-the Potentials and Limitations. Manchester: St. Jerome Publishing.

[17] Reiss, K., \& Vermeer, J. H. (2014). Towards a general theory of translational action: Skopos Theory explained. Routledge.

[18] Saldanha, G., \& O'Brien, S. (2016). Research methodologies in translation studies. Perspectives,24. https://doi.org/https://doi.org/10.1080/0907676x.2015.1109906

[19] Temple, B., \& Young, A. (2004). Qualitative Research and Translation Dilemmas. Qualitative Research, 4(2), 161-178.

[20] Tu, Z. (2000). English reading and translation for electrical engineering. Shanghai Foreign Language Education Press.

[21] Tu, Z., \& Xi, J. (2002). Translation of English for Special from the Perspective of English for Electric Power. Journal of Shanghai Institute \& Electric Power.

[22] Vermeer, H. J. (1987). What Does It Mean to Translate? Indian Journal of Applied Linguistics, 13(2), 25-33.

[23] Vermeer, H. J. (1989). Skopos and commission in translational action. Routledge.

[24] Vinay, J. P., Darbelnet, J. (1995). Comparative stylistics of French and English: A methodology for translation (Vol. 11). John Benjamins Publishing.

[25] Wang, H. (2016). Analysis of the Electric English Terms and Reform of Translation. Journal of Hunan City University(Natural Science), 25(5), 297-298.

[26] Yang, M., \& Gao, J. (2015). Characteristics and translation skills of electrical English terms. Jiangsu Foreign Language Teaching and Research, 4, 85-87.

[27] Fan, \& Zhongying. (1990). Some remarks on the criteria of translation. Babel, 36(2), 97-110. 